\title{
Study of Image Harris detector Based on Regular Grid
}

\author{
Yang Dong ${ }^{1, a}$ Jingguo $\mathrm{LV}^{1, \mathrm{~b}}$ and Xian Zhao ${ }^{1, \mathrm{c}}$ \\ ${ }^{1}$ Beijing University of Civil Engineering and Architecture,Beijing 100000, China; \\ aljcdy163ggyx@163.com, 'blvjingguo@bucea.edu.cn, czhaoxian@bucea.edu.cn
}

Keywords: corner detection,the Harris corner detection algorithm,regular grid

\begin{abstract}
Corner detection is a kind of important method for image feature extraction. This paper analyzes the Harris corner detection algorithm, due to the phenomenon that original algorithm of corner response functions are gathered is existed in the high resolution image detection, this article solves the distribution is not balanced through the establishment of regular grid. At the same time, good basic units for the subsequent image matching are provided .The experimental results prove that the improved algorithm greatly improves the image corner detection and matching accuracy.
\end{abstract}

\section{Introduction}

In the field of image processing, image matching is one of the key technologies. And feature point extraction provides a good basis for image matching. Therefore, feature extraction is very important in image matching[1].The methods of feature points extraction mainly have Harris operator, Moravec operator, Forstner operator, Susan operator,and so on. Harris corner detector is more suitable, and the feature points are stable, robust and consistent with the visual angle. And the calculation is moderate,which is commonly used.However,for lack of texture features, the relative concentration of the intensity of the image, the feature points of Harris corner detector is less, and the feature points of texture rich regions are clustered.These bad phenomena are not conducive to the follow-up work or the results are very difficult to satisfy.

This paper selects the Harris operator to extract the feature points of the buildings on the ground, because it has a good effect. First of all, this paper introduces the Harris operator, then the image is divided into plurality of uniform grids, according to the threshold in the each grid,the corresponding feature points are extracted in each grid. Finally, the experimental results are analyzed and summarized.

\section{Harris detector based on regular grid}

\subsection{Harris corner detector algorithm[2].}

Harris corner detector is based on the local auto correlation function of the signal. The principle of Harris algorithm is to design a local window. When the window is moving along all directions, the average energy of the window is changed. If the pixel is in the inner region, the average energy change is small in any direction;If the pixel is located in the edge region, the average energy intensity changes along the edge direction, and the average energy changes largely in the vertical direction; If the pixel is a corner, the average energy change along the direction of the random direction is very large.Based on the analysis of the feature properties, Harris and Stephens[3] are inspired by the work of H.Moravec, and the $\mathrm{f}(\mathrm{I})$ is transformed into $\mathrm{M}$ (I).

$$
\begin{gathered}
M(x, y)=\left[\begin{array}{cc}
\sum_{w} I_{x}(x, y)^{2} & \sum_{w} I_{x}(x, y) I_{y}(x, y) \\
\sum_{w} I_{y}(x, y) I_{y}(x, y) & \sum_{w} I_{y}(x, y)^{2}
\end{array}\right]=\left[\begin{array}{ll}
A & C \\
C & B
\end{array}\right] \\
R=\operatorname{det}(M)-k \times \operatorname{trace}(M)^{2}
\end{gathered}
$$

Harris defines the response function to detect the corners and edges of the image.Among them, Ix and Iy, respectively, present the gradient of the pixel in the horizontal direction and the vertical direction.Det(M) and trace (M) are the determinant and trace of M respectively.K is constant, whose 
average value is 0.04 to 0.06 . R represents the interest value of the corresponding pixel points in the image.The threshold value $\left(T_{0}\right)$ is set to $R$. If the interest value of a pixel is biggest in the neighborhood,and it is greater than the threshold $\left(\mathrm{T}_{0}\right)$, then the pixel corner response function CRF (Corner Response Function) is called the corner point, the corresponding interest value is called the corner angle value, and then the local Maximum function is chosen to avoid the improper corners.Finally, the feature points can be detected.

\subsection{Extract feature points in a regular grid.}

Harris algorithm is a widely used in point feature extraction , only referenced to the gray level of a differential[4] and filtering.The speed and efficiency of detecting corner points can be ensured effectively.For all local extreme points ranking, according to the need to extract the characteristics of the point.And according to the required number of feature points, all the local extreme points are ranked to extract the characteristics of the points for the need.The image has a good robustness, such as rotation, gray level change, noise and viewpoint. But because of the difference of gray changes of the whole image, using Harris algorithm for image corner extraction needs to except these maximum values[5,6]. The effect of corner extraction is entirely dependent on the threshold.The threshold is too large to detect the corner points, resulting in the loss of some corner points.If the threshold is too small ,the corner points will not be extracted from the image points, resulting in angular redundancy or corner clustering.So as to extract the image feature points in uneven texture image, the distribution of the feature points of the image is not uniform. In large areas, the same color may result in the distribution of feature points centrally.

The feature points are extracted using Harris algorithm, according to the initial threshold, certain feature points are automatically extracted. Then the original pixels are divided into rectangular elements with the same size,who is named a regular grid.The feature points are extracted from each rectangular element as the basis of data processing. The feature points of each rectangular grid extraction, Harris corner response function values are sorted in descending order.The average value of corners response function value in each grid is set the threshold ,which is named T.The feature points of more than $\mathrm{T}$ are extracted, and the feature points of less than $\mathrm{T}$ are deleted. If the statistical characteristics is not good, it is still a problem that there are still too many or too little points.It is very necessary to continue to iterate the above steps until a certain number of feature points are removed.In each rectangular grid, feature points extracted by Harris algorithm is controlled in a certain number range, to ensure the extraction of feature points evenly distributed, which has no feature points crowded.The whole algorithm has good robustness. Experiments show that the proposed algorithm has some improvement on the speed and accuracy of the existing feature point matching algorithm.

\section{Experimental result analysis}

In order to verify the effectiveness of Harris detector based on regular grid in image corner detection, the algorithm is implemented, and compared the results with previous algorithms.

Figure 1 shows using the original Harris algorithm, whose threshold is set relatively large,extracts feature points.Figure 2 uses the original Harris algorithm to extract feature points, however the threshold is set relatively small. In Figure 1, part of the corners are not shown in the windows, the bricks and other corner places,just because the threshold is too large.In Figure 2, due to the low threshold,the image that the gray levels change in small regions produces a phenomenon feature points are clustered, which is resulted in redundancy in a certain degree, such as in the wall, trees, body and other relatively simple area of the texture of the phenomenon of corner redundant.For example, on the wall, trees and other regions whose texture is relatively simple where is the redundancy phenomenon of the corners.It can be seen from the Figure 3,whose corner points are extracted by using the algorithm of Harris detector based on regular grid.Compared with the original algorithm,the image is divided into regular grid to extract the corner,the feature points extracted with this algorithm are evenly distributed in the whole image. The extraction of corner points are reduced in which the texture is not rich in the areas.In some corners, such as windows, 
brick corners, etc, were not extracted from original corner detection algorithm.However, this time corner points are clearly detected .

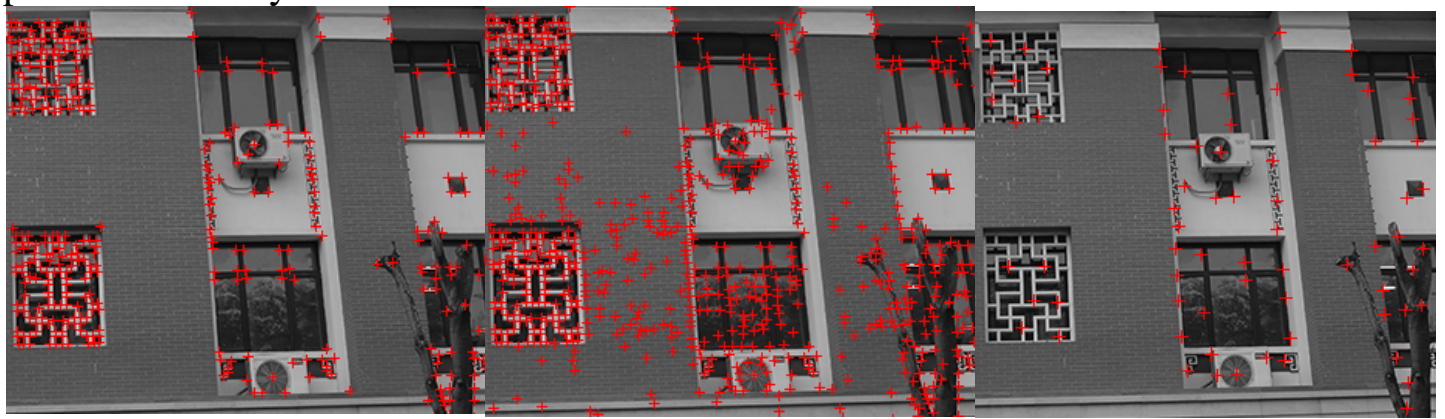

Fig.1.the threshold is larger Fig. 2.the threshold is smaller Fig.3 based on regular grid

It can be seen from the figure 4, the images is to be matched with the original algorithm for corner extraction. The distribution of the feature points is dense, not conducive to image matching. Then we use this algorithm to extract the corner of the two images, and remove the corner points of the low contrast feature points, and the effect is shown in Figure 5. At last, the corner points extracted from Figure 5, are carried out the image matching. The matching effect of Figure 6 is obtained.As we can see from the graph, matching is successful.The feature points with the uniform distribution extracted from the essay's algorithm provide a good basis for the matching.

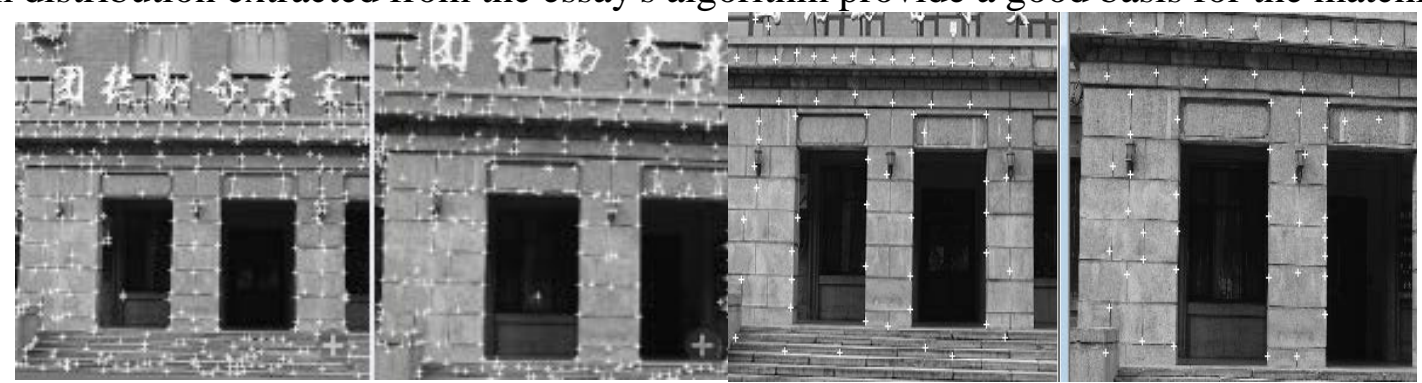

Fig. 4 the Harris detector

Fig.5 Harris detector based on regular grid

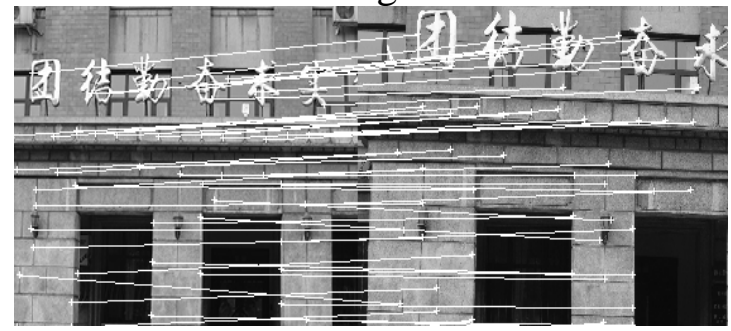

Fig.6 The matching image from Harris detector based on regular grid

\section{Conclusion}

Finally, experiments show that the extraction of feature points by using the Harris detector based on regular grid,which controls the number of feature points,ensuring uniform distribution characteristic, provide good matching primitives.The experimental effect is good.And it can provide relatively stable control points for the following work. The whole algorithm also has strong stability, noise immunity and wide application, so it has a certain application prospect and significance.

\section{References}

[1]. Zhanlong Yang, Baolong Guo . Research on image registration and mosaic technology based on feature points [D]. Xi'an: Xi'an Electronic and Science University, 2009

[2]. Shengli Cai , Huiqing Zhang. Measurement and control of [J]. based on Harris corner detection, 2011,19 (1): 30-32.

[3]. Harris C G , St ephens M J. A Combined Corner and Edge Detect or. Proceedings Fourth Alvey Vision Conference, Manchester,1988: 147 151 
[4]. Xiaojing Guo, Yi Fang. Zhiyu Li .The image corner detection method of the [D]. Qingdao: Qingdao University, 2009

[5]. Jia Wei. Study on the Harris feature point extraction algorithm for [J]. computer knowledge and technology, 2010, 32 (6): 9089-9090.

[6]. Yanjie Hou, Jie Cao. A method of image mosaic based on Harris corner feature [J].information research ,2010,36(10):23-25.DOI:10.3969/j.Issn.1674-4888.2010.

10.007 . 